\title{
OBSTÁCULOS NA ASSISTÊNCIA DE ENFERMAGEM À PESSOA COM DIABETES NA HOSPITALIZAÇÃO ${ }^{1}$
}

\section{OBSTACLES IN NURSING CARE FOR PEOPLE WITH DIABETES DURING HOSPITALIZATION}

\author{
Caroline Rocha Batista Barcellos ${ }^{2}$ \\ Juliana Graciela Vestena Zillmer ${ }^{3}$ \\ Bárbara Resende Ramos ${ }^{4}$ \\ Ana Laura da Silva Barragana Vera ${ }^{5}$
}

\begin{abstract}
Resumo: Descrever os obstáculos vivenciados pelos enfermeiros na assistência à pessoa com diabetes mellitus na hospitalização. Pesquisa qualitativa com 15 enfermeiros de um Hospital de Ensino do Rio Grande do Sul. A coleta de dados ocorreu entre outubro a novembro de 2019, produzida por meio de entrevista semiestruturada, posteriormente organizados mediante programa Etnograph v6 e, submetidos à análise de conteúdo. Foram construídas seis categorias que correspondem aos seguintes obstáculos: entendimento e conhecimento insuficiente, dificuldade de aceitar viver com a doença e tratamento, complexidade de modificar hábitos e estilo de vida, automanejo dos medicamentos e oferta e distribuição da dieta no hospital. Os obstáculos na assistência de enfermagem no hospital ajudam a apreender a realidade de um contexto complexo, que envolve cuidados prévios que levaram a internação, cuidados durante a hospitalização e a planejar a alta hospitalar.
\end{abstract}

Palavras-chave: Diabetes Mellitus; Enfermeiros; Hospitalização; Pesquisa Qualitativa.

\begin{abstract}
To describe the difficulties experienced by nurses in assisting people with diabetes mellitus during hospitalization. Qualitative research with 15 nurses from a Teaching Hospital in Rio Grande do Sul. The data collection took place between October and November 2019, produced through semi-structured interviews, later organized using the Etnograph v6 program and submitted to content analysis. Six categories were constructed that correspond to the following obstacles: insufficient understanding and knowledge, difficulty accepting to live with the disease and treatment, complexity of changing habits and lifestyle, self-handling of medications and supply and distribution of the diet in the hospital. The obstacles in nursing care in the hospital help to apprehend the reality of a complex context, which involves previous care that led to hospitalization, care during hospitalization and planning for hospital discharge.
\end{abstract}

Keywords: Diabetes Mellitus; Nurses; Hospitalization; Qualitative research.

\footnotetext{
${ }^{1}$ Estudo extraído do Trabalho de Conclusão de Curso "Práticas de cuidado ao paciente com diabetes mellitus hospitalizado: o que dizem os enfermeiros?" apresentado à Faculdade de Enfermagem da Universidade Federal de Pelotas em 2019.

${ }^{2}$ Enfermeira pela Universidade Federal de Pelotas (UFPel). Residente no Hospital Regional Hans Dieter Schmidt, Joinville, Santa Catarina, Brasil. E-mail: caroline.rbb@gmail.com

${ }^{3}$ Doutora em Enfermagem pela Universidade Federal de Santa Catarina (UFSC). Universidade Federal de Pelotas (UFPel), Pelotas, Rio Grande do Sul, Brasil. E-mail: julianavzillmer@gmail.com

${ }^{4}$ Mestre em Ciências da Saúde pela Universidade Federal de Pelotas (UFPel). Hospital Escola da Universidade Federal de Pelotas/Empresa Brasileira de Serviços Hospitalares (UFPel/EBSERH), Pelotas, Rio Grande do Sul, Brasil. E-mail: barbararesende.ramos@gmail.com

${ }^{5}$ Acadêmica de Enfermagem pela Universidade Federal de Pelotas (UFPel). Universidade Federal de Pelotas (UFPel), Pelotas, Rio Grande do Sul, Brasil. E-mail: anasbv99@gmail.com
} 


\section{Introdução}

A diabetes mellitus é uma emergência de saúde pública em nível mundial. Estimase que até 2019, 463 milhões de pessoas no mundo viviam com a doença o que gerou numerosos gastos para os países (BOMMER et al., 2018; INTERNATIONAL DIABETES FEDERATION, 2019). O Brasil registra o maior número de adultos com diabetes, sendo 16,8 milhões e, estima-se que também seja o País com o terceiro maior número de crianças e adolescentes com diabetes tipo 1 no mundo, ficando atrás apenas dos Estados Unidos e Índia. Ainda se evidencia o contínuo aumento de pessoas sem diagnóstico de diabetes, a maioria do tipo 2 , revelando uma porcentagem superior a $50 \%$ que traz a necessidade de uma atenção apropriada a fim de diagnosticar e, dar continuidade ao tratamento a essa população (INTERNATIONAL DIABETES FEDERATION, 2019).

As complicações prevalentes que levam os pacientes com diabetes à internação são hiperglicemia, hipoglicemia e cetoacidose diabética (ARRUDA; SCHMIDT; MARCON, 2018), contudo a hiperglicemia é a principal ocorrência relacionada à diabetes nos hospitais (BOGUN; INZUCCHI, 2013). No diabetes tipo 2, isto decorre da baixa aceitação terapêutica ou a falha dela, principalmente com tempo de diagnóstico maior ou igual a 10 anos, presença de comorbidades, não realização de consultas no último um ano e, dificuldade de acesso a profissionais especializados, entre outros motivos (ARRUDA; SCHMIDT; MARCON, 2018).

Deste modo, é necessário que ocorra o gerenciamento cuidadoso desses pacientes hospitalizados a fim de reduzir o tempo de internação e, necessidade de readmissão, além de considerar uma transição cuidadosa da assistência do hospital para o nível ambulatorial (AMERICAN DIABETES ASSOCIATION, 2020). As diretrizes de tratamento para pessoas com diabetes incluem ações complexas e específicas e, as pessoas que vivem com a doença têm dificuldades em autogerenciar a sua condição de saúde para alcançar o controle da glicemia (SOCIEDADE BRASILEIRA DE DIABETES, 2020; BLASCOBLASCO; PULG-GARCIA; PIAY et al., 2020). Estudo descreve que os pacientes com diabetes mellitus 2 consideram difícil a realização do tratamento, principalmente em relação a alimentação, prática de exercícios físicos e uso de medicação (MWILA; BWEMBYA; JACOBS, 2019).

Estudos apontam barreiras na comunicação enfermeiro-paciente que interferem diretamente na assistência realizada (MULDER et al., 2015; MWILLA; BWEMBYA; 
JACOBS 2019). Os enfermeiros ao observarem a desistência do tratamento pelos pacientes perdem a motivação para falar sobre autogestão e, passam a aconselhá-los de forma geral. Isto resulta em um cuidado ainda mais insatisfatório, uma vez que a aceitação do processo é dificultosa e, os pacientes necessitam de uma atenção individual, de acordo com suas condições físicas, emocionais, socioeconômicas e culturais para darem continuidade no domicílio (MULDER et al., 2015; MWILLA; BWEMBYA; JACOBS, 2019).

O enfermeiro desempenha papel fundamental na assistência à saúde da pessoa com diabetes durante a hospitalização e, está principalmente ligada a prevenção de complicações, recuperação e, promoção da saúde. Contudo, esse trabalho demanda ações educativas no âmbito hospitalar, o qual é um processo lento devido os obstáculos que os profissionais encontram, por exemplo, a sobrecarga de atividades, o espaço físico e recursos materiais inadequados (ARRUDA; SILVA, 2020). Diante do exposto, foi construída a seguinte questão de pesquisa: quais os obstáculos vivenciados pelos enfermeiros na assistência à pessoa com diabetes durante a hospitalização? Para respondê-la, o estudo teve como objetivo descrever os obstáculos vivenciados pelos enfermeiros na assistência à pessoa com diabetes mellitus durante a hospitalização.

\section{Método}

Pesquisa qualitativa realizada em um hospital de ensino na Região Sul do Rio Grande do Sul, Brasil. Tal hospital foi escolhido por ter vínculo com o Centro de Diabetes e Hipertensão, referência em atendimento à pessoa com diabetes na Região e, ambos estarem sob a responsabilidade de uma Universidade Pública. O trabalho de campo foi desenvolvido entre setembro a outubro de 2019, pela primeira autora, discente de enfermagem, devidamente capacitada e, sob supervisão e orientação da segunda e terceira autora que apresentam experiência nessa área do conhecimento e na abordagem de pesquisa. A quarta autora, discente, ficou responsável por organizar e gerenciar as transcrições e posteriormente os dados no programa para construção do estudo.

Os participantes do estudo foram enfermeiros das Unidades da Rede de Urgência e Emergência I, II e III, Clínicas Médica e Cirúrgica, mediante amostragem do tipo intencional. As Unidades da Rede de Urgência e Emergência surgem com a reformulação da Política Nacional de Atenção às Urgências em 2011, onde se instituiu a Rede de Atenção às Urgências no Sistema Único de Saúde, visando promover ampliação e 
qualificação dos atendimentos prestados a população que necessita recorrer os serviços de saúde em casos de urgência e emergência, como no caso dos leitos de retaguarda em ambiente hospitalar (BRASIL, 2011). No hospital de realização da pesquisa, estas unidades correspondem às enfermarias clínicas que internam pacientes provenientes principalmente do pronto socorro municipal, sendo também as Unidades onde intermam pacientes com diabetes mellitus seja como causa da internação por agudização da mesma ou como comorbidade secundária. Estes foram selecionados a partir dos seguintes critérios: ser enfermeiro da unidade há mais de seis meses e, que tivessem vivenciado à assistência à pessoa com diabetes mellitus. Quanto aos critérios de exclusão, foram considerados, enfermeiros em licença saúde ou férias.

Para obter o total de enfermeiros nas referidas unidades, a pesquisadora contou com o apoio da gerência de enfermagem da Instituição mediante o fornecimento de uma lista contendo os profissionais que estariam atuando no período de coleta, contemplando os três turnos de trabalho. No início da pesquisa, o tamanho da amostra não havia sido definido, entretanto, com base na revisão da literatura de pesquisas com abordagem qualitativa, considerou-se incluir pelo menos 10 participantes. No período do trabalho de campo havia um total de 30 enfermeiros nas referidas Unidades, sendo que destes participaram do estudo 15 enfermeiros que atenderam os critérios de inclusão e concordaram participar do estudo. Treze eram mulheres e dois homens. Ao analisar as informações obtidas, considerou-se que se tratava de um tamanho amostral adequado devido à riqueza de dados que permitem responder à questão de pesquisa e compreender o objeto do estudo. Além disso, com o avançar da realização das entrevistas havia dados que se repetiam. Assim, considera-se que a amostra refletiu "em quantidade e intensidade, as múltiplas dimensões de determinado fenômeno e busca a qualidade das ações e das interações no decorrer de todo o processo" (MINAYO, 2017, p.10).

A técnica de coleta de dados utilizada foi a entrevista semiestruturada, sendo elaborado um guia contendo perguntas abertas e fechadas considerando o objetivo da pesquisa. As entrevistas foram desenvolvidas individualmente, em horários previamente definidos com os participantes e, realizadas em uma sala reservada do hospital, conforme escolha do entrevistado. A primeira pergunta da entrevista foi aberta, sendo ela: "Podes me contar sobre sua experiência ao cuidar de pessoas com diabetes durante a hospitalização?", posteriormente seguida de outras perguntas que gradativamente tinham a finalidade de aprofundar o tema. As 15 entrevistas foram gravadas, resultando em 
arquivos de áudios e, logo foram transcritos na íntegra pela primeira autora, sendo revisadas pela mesma, com escuta dos áudios gravados e leitura do texto escrito.

Para organização e gerenciamento do conjunto de dados das entrevistas foi utilizado o Programa Ethnograph V6 em sua versão demonstração. Este conjunto de dados foi analisado mediante análise de conteúdo conforme proposta de Laurence Bardin (BARDIN, 2016). A análise inicialmente consistiu na leitura dos dados a fim de atingir a imersão e, obter uma compreensão do todo por parte da primeira autora. Posteriormente, procedeu-se à leitura linha a linha, até geração de códigos. Em seguida, os códigos foram comparados e, fragmentos de texto selecionados para identificação dos temas, que posteriormente deram origem a categorias. Foram desenvolvidos 24 códigos, e destes foram construídas seis categorias.

A pesquisa foi aprovada pelo Comitê de Ética em Pesquisa de uma Universidade pública sob CAAE de número 19579519.8.0000.5317, sendo seguidos preceitos éticos da Resolução 466/12 (BRASIL, 2012) e da Resolução 564/17 do Código de Ética de Enfermagem (COFEN, 2017). Além disso, os participantes do estudo assinaram o Termo de Consentimento Livre e Esclarecido. A fim de garantir o anonimato, os participantes foram identificados pela letra "E” para Enfermeiro, seguido de números para diferenciálos conforme a sequência em que as entrevistas foram realizadas.

\section{Resultados e Discussão}

A partir da análise dos dados foram construídas as seguintes categorias que descrevem os obstáculos vivenciados pelos enfermeiros para desenvolver a assistência à pessoa com diabetes na hospitalização: "Uns se cuidam, outros não": entendimento e conhecimento insuficientes sobre o diabetes; "A gente vê que tem essa questão de aceitação": a complexidade do adoecimento; "Tudo que exige uma mudança comportamental é complexo": alimentação no manejo do tratamento; "Então essa é uma dificuldade também, perceber a adesão do paciente ao tratamento": manejo dos medicamentos; "O entendimento da própria família é bem difícil"; "Mudança na rotina das refeições": a dieta no hospital". 


\title{
3.1 "Uns se cuidam, outros não": entendimento e conhecimento insuficientes sobre
}

\section{o diabetes}

Para os enfermeiros, há obstáculos que correspondem principalmente, a dificuldade de entendimento e, conhecimento insuficiente do paciente acerca da doença e tratamento para o diabetes. Este entendimento e conhecimento pode variar de acordo com o contexto sociocultural, e nível de escolaridade, associado ao acesso de informação nos serviços de saúde. Em decorrência disso, poderá resultar em complicações agudas, por exemplo, a descompensação daglicemiae, recorrentes internações no hospital. Tais achados podem ser identificados nos seguintes relatos:

\begin{abstract}
$\mathrm{Ah}$, tu vais conversar com as pessoas que tem menor poder aquisitivo assim, $\mathrm{e}$ até as que têm até certo nível de compreensão, de escolaridade assim, sabem um pouco melhor, eles não entendem bem o que é o diabetes. Eles não entendem as implicações relacionadas ao diabetes, então é bem, em função disso é difícil tu manter, tu podes tentar cuidar. Mas, a gente precisa da participação do paciente nesse processo, porque ele não é uma mão de via única, então o paciente precisa participar do processo de seu próprio cuidado. A dificuldade é, não só em especifico o paciente diabético, acho que a maior, a maioria dos pacientes em geral, é a questão do nível de compreensão da própria doença (E09).
\end{abstract}

A maior dificuldade é a questão da compreensão mesmo, das coisas que ele utiliza, eles utilizam [alguns alimentos] há bastante tempo e, que faz mal. Mas ele não sente, tu entendes? Ele pode fazer, provocar uma hiperglicemia, mas ele não está sentindo nada, não tem sintoma naquele momento e, a falta de sintoma, ele acaba associando como se estivesse tudo bem (E010).

Isso é comum a todas as doenças crônicas. Difícil, tu por toda vida, ser uma pessoa muito regrada, uma pessoa extremamente cuidadosa. As doenças crônicas têm essa característica, as pessoas em alguns momentos relaxam, desanimam, querem comer, querem fazer aquilo que não pode. Então isso é uma dificuldade, muitos pacientes chegam descompensados ou acabam precisando fazer a insulina aqui, todos os dias ou em quase todos os horários, mas por questões deles próprios, assim. Que não tinham um cuidado já, préhospitalar, pré-internação (E06).

A dificuldade de compreender a doença e a progressão do adoecimento foi justificada pelos enfermeiros, uma vez que, os pacientes não apresentam sintomas, tendo a constante sensação de que está tudo bem e, por isso não desenvolvem o autocuidado. A diabetes mellitus foi descrita também pelos profissionais como sendo uma doença "silenciosa", em que os pacientes nem sempre identificam e ou sentem que há algo estranho ocorrendo no corpo e, com o passar do tempo, desencadeará sinais e sintomas que correspondemao avanço da enfermidade. Em decorrência disso, seguem realizando determinadas práticas, com maior ênfase ao consumo de alimentos que alteram a glicemia, e consequente surgimento de complicações que por diversas vezes, não são percebidas pelo paciente como relacionadas a diabetes. 
Estudo de revisão desenvolvido na América Latina e Caribe identificou a importância do conhecimento do paciente e do profissional para o gerenciamento adequado do tratamento e manejo dos efeitos colaterais (BLASCO-BLASCO; PULGGARCIA; PIAY et al., 2020). A dificuldade de entendimento e conhecimento insuficiente dos pacientes sobre a diabetes e o tratamento, identificada no presente estudo, não é somente uma realidade vivenciada no cenário brasileiro. Estudo desenvolvido nos Países Baixos identificou que os profissionais de enfermagem também encontram problemas na falta de comunicação entre eles e os pacientes, algo que dificulta o processo de entendimento a respeito da doença (MULDER et al., 2015).

Uma comunicação inadequada entre enfermeiro, paciente e família pode dificultar a assistência e ocorrer resistência, pois se vêem obrigados a mudar seus hábitos de vida rigorosamente sem que antes tenham entendimento suficiente sobre os benefícios na condição de saúde. Somando-se a isto, há profissionais de saúde que ao acompanhar e assistir a pessoa com diabetes estabelecem uma relação autoritária realizando orientações de forma impositiva e proibitiva (GAMA; GUIMARÃES; ROCHA, 2017). Esta forma de relação é apontada como imprópria pois dificulta o cuidado e autogestão do tratamento proposto (GAMA; GUIMARÃES; ROCHA, 2017; PELTOLA; ISOTALUS; ÅSTEDTKURKI, 2018).

Em contrapartida a estas atitudes, estudo aponta que construir uma relação interpessoal respeitosa entre profissional-paciente resulta em boas experiências de cuidado e autogerenciamento (PELTOLA; ISOTALUS; ÅSTEDT-KURKI, 2018). Uma das estratégias para qualificar a comunicação e essa relação é promover um processo de negociação, a fim de fornecer informações e pactuar o que é possível realizar enquanto mudanças de hábitos e estilo de vida. Este processo de negociação possibilita que a pessoa com diabetes participe da tomada de decisão sobre seus cuidados e seja responsável pelo gerenciamento do tratamento e seu autocuidado (PELTOLA; ISOTALUS; ÅSTEDTKURKI, 2018; GAMA; GUIMARÃES; ROCHA, 2017; PERES et al., 2007).

Associado a comunicação inadequada é necessário considerar o letramento das pessoas com diabetes, o qual pode estar relacionado com a dificuldade de entendimento e, conhecimento insuficiente. Estudo de revisão aponta que o grau de letramento limitado de pacientes podem resultar em uma menor adesão ao tratamento (MARAGNO; LUIZ, 2016). Desse modo, um menor grau de letramento pode impossibilitar o adequado autocuidado e gerenciamento do tratamento, e prejudicar a compreensão e aquisição de 
práticas de saúde, assim como aumentar o surgimento de complicações e incapacidades (ROCHA et al., 2019).

Estudo de revisão aponta que melhorar a alfabetização em saúde de pessoas com diabetes pode diminuir a carga emocional da doença e aumentar o conhecimento (BLASCO-BLASCO; PULG-GARCIA; PIAY et al., 2020). Além disso, avaliar o nível de letramento em saúde pode ser uma importante estratégia para direcionar as orientações de cuidado. Ainda, durante a hospitalização é necessário desenvolver o uso de linguagem específica e estratégias individualizadas a cada paciente e família, considerando a fase do ciclo de vida e seu contexto sociocultural (MARAGNO; LUIZ, 2016; ROCHA et al., 2019).

\section{2 "A gente vê que tem essa questão de aceitação": a complexidade do adoecimento}

A dificuldade de aceitação da diabetes e do tratamento pelos pacientes foi apontada como um obstáculo para os enfermeiros. Eles apontaram que a diabetes por ser uma doença crônica implica na adoção de cuidados contínuos e restrições que se estendem ao longo da vida, o que acarreta maior dificuldade de aceitação da nova condição de saúde. Entre as restrições há necessidade de mudanças, principalmente no estilo e hábitos de vida, com maior ênfase, em substituir alimentos ou extingui-los quando necessário para controle da glicemia. Por estes motivos, os pacientes vão "descuidando" ao longo do tempo e, até mesmo abandonando, em parte ou todo, o tratamento. Tais achados podem ser identificados no seguinte excerto:

Então muitas vezes a gente vê que tem essa questão de aceitação e param por conta própria [o tratamento] [...]. Então eu acho que muitas vezes é a aceitação, primeiramente do paciente, ele entender o caso, ele entender que ele vai ter que ter um controle diretamente de uma dieta, e esse controle da dieta tem que ser, ah rígida. [...]. É importante o paciente entender que a doença é uma doença crônica, que se não tratada pode levar a várias complicações, ele tem que entender isso e querer se ajudar (E08).

Os enfermeiros descreveram que há maior dificuldade em aceitar a doença e o tratamento quando os pacientes são adolescentes e jovens. Estes internam em decorrência de complicação do diabetes tipo 1 e, alguns recebem o diagnóstico da doença durante a hospitalização. Contudo, as frequentes internações destes jovens e adolescentes devido às complicações agudas foram descritas como sendo resultantes por quererem viver uma vida "normal". Isto pode ser evidenciado nos seguintes relatos: 
Eu tive um menino aqui de 16 anos que ele já teve cetoacidose milhares de vezes. Internou por esse motivo milhares de vezes, porque ele é um paciente adolescente que quer viver uma vida normal (E02).

Especialmente [é mais difícil por ser mais jovem] com aquele paciente que é diabete tipo 1, porque muitos deles que a gente já teve aqui eles internam e, eles têm dificuldade até de aceitação. Às vezes ele passa toda juventude mal e não descobre e, quando descobre, ele vem parar aqui [no hospital]. Então ele tem muita dificuldade de aceitação, tem todo processo de alimentação, eles não aceitam (E03).

Percebe-se que os profissionais enfermeiros têm o entendimento que a diabetes promove um contexto de mudança na vida da pessoa, não sendo mais possível que o mesmo tenha uma vida "normal". Desta forma, destacaram a dificuldade enfrentada pelos jovens e adolescentes que se encontramem uma fase do ciclo de vida de descobertas e de planejar o futuro, porém vivenciam limitações ocasionadas pela diabetes e as frequentes internações.

Em estudo desenvolvido por Blasco-Blasco e colaboradores (2020) a dificuldade de aceitar o diagnóstico e se identificar como "paciente" foi constatada como uma barreira significativa para o cuidado do diabetes. Receber o diagnóstico é considerando uma fase estressante para a família, principalmente quando se trata de crianças, adolescentes, somado à própria internação hospitalar (ORTIZ et al., 2017). Após o diagnóstico, a pessoa com diabetes vivencia uma diversidade de emoções, como ansiedade, medo, nervosismo, revolta e aflição, que resultam em distintas respostas emocionais entre aceitação e resistência (RODRIGUES; LIMA; SANTOS, 2015).

Desenvolver a assistência a uma pessoa com diabetes requer do enfermeiro habilidades e competências que ultrapassem a concepção biomédica. O profissional necessita acolher a pessoa e sua família considerando os aspectos sociais e culturais e, mediante uma relação dialógica, fornecer informações e empoderá-lo para que gradativamente consiga gerenciar seu próprio tratamento. Para isso, estudos apontam a educação em saúde como uma importante ação desenvolvida pelo enfermeiro às pessoas com diabetes durante a hospitalização (NIKITARA et al., 2019; ARRUDA; SILVA 2020).

A educação em saúde é imprescindível, devido, principalmente, ao desconhecimento das complicações que a diabetes pode ocasionar (ARRUDA; SILVA, 2020). Além de possibilitar o empoderamento e promover a continuidade do cuidado no domicílio (NIKITARA et al., 2019). A fim de desenvolver um melhor entendimento da diabetes e tratamento, prevenir complicações, promover uma melhor qualidade de vida assim como, gerenciar o próprio tratamento é necessário atentar-se para estratégias que 
considerem a fase do ciclo de vida na qual a pessoa com a doença se encontra. Para as crianças, por exemplo, recomenda-se que a partir dos sete anos, elas assumam progressivamente suas atividades diárias desde que supervisionadas por adultos capacitados, assim como, possam ser instruídas sobre a fisiopatologia da diabetes de forma simplificada. O uso da tecnologia como jogos e redes sociais, além do manuseio de bonecos e fantoches ajudam na compreensão de forma mais didática (ORTIZ et al., 2017)

$\mathrm{Na}$ abordagem para jovens, é necessário o auxílio da família buscando não o sobrecarregar, porém o incentivar para a autonomia, de modo que seja protagonista do seu próprio cuidado. É importante que, ao mesmo tempo, não se sintam sozinhos nesta mudança de hábitos, uma vez que se encontram em uma fase de transição para a vida adulta na qual lidam com diversas transformações físicas, emocionais e sociais (ORTIZ et al., 2017).

Em relação aos idosos, atividades educativas em grupo resultam em melhorias nos aspectos de alimentação e cuidados com os pés. Estes achados são significativos, uma vez que a troca de informações entre eles é benéfica para a aprendizagem do autocuidado. Ademais, os idosos podem compartilhar e passar as explicações para outros indivíduos com diabetes, disseminando o conhecimento para outros e reforçando-os para si (MARQUES et al., 2019).

\section{3 "Tudo que exige uma mudança comportamental é complexo": alimentação no manejo do tratamento}

Para os enfermeiros, a alimentação mostrou-se como o principal obstáculo vivenciado pelas pessoas com diabetes. Isto porque exige mudanças nos hábitos e estilo de vida para adoção de uma alimentação saudavél. Para os participantes, o paciente necessita compreender e diferenciar os alimentos que podem ser utilizados e, aqueles que provocam alterações na glicemia. Descrevem como exemplo disso o que ocorre no hospital quando hospitalizados os pacientes comem escondido determinados alimentos, com maior ênfase a doces e lanches, trazidos pelos familiares.

O comer doce foi descrito pelos enfermeiros como uma prática universal, uma vez que, toda pessoa gosta e para continuar com esta prática eles manejam os medicamentos que fazem uso. A alimentação foi descrita como responsável por desencadear e acelerar 
as complicações, por exemplo, a amputação de membros. Isto pode ser evidenciado nos seguintes relatos:

\begin{abstract}
Uma outra dificuldade, é do próprio paciente, de se adequar as restrições alimentares, mas isso também é inerente da pessoa, da pessoa humana, não é uma dificuldade propriamente do nosso setor, as pessoas, é bom comer doce. É bom comer carboidrato, e todo mundo gosta e os pacientes também, não são diferentes. Então, alguns deles quando podem comem escondido, isso a gente observa, isso é muito comum, muito frequente. Então essa é uma dificuldade também, perceber a adesão do paciente ao tratamento, principalmente a dieta. As medicações até eles não resistem muito né, tanto é que tem pacientes que dizem, comentam com a gente "Ah, prefiro poder comer de tudo e fazer minha medicação". [...] As pessoas preferem se medicar, mas terem uma liberdade um pouco maior em relação a dieta, essa é uma dificuldade conforme eu te falei, inerente do ser humano, não é uma particularidade dos nossos pacientes (E06).

Como é que fica sem circulação nenhuma e fica com aquela toda deficiência, e aquele paciente perde o membro, então isso é complicado para ele aceitar. A gente já teve paciente jovem aqui sem perna, sem braço, aqueles pacientes que não se cuidam, então isso é difícil (E03).

Eles dizem "Ah, eu comi um lanchezinho que me trouxeram [a família trouxe]". E aí tu vês, tudo aquilo podia ser perdido, todo trabalho que foi feito [tratamento], podia ser perdido, porque ele não aceitou receber somente a dieta que o hospital provia (E08).
\end{abstract}

As mudanças alimentares foram descritas como sendo as mais complexas para serem implementadas pelos pacientes com diabetes (MARTINS; MATTOS; DIERCKS, 2020; REIS et al., 2020). A complexidade decorre porque para os pacientes são vistas com um caráter restritivo e não como uma parte importante e passível de flexibilidade e adaptação (MARTINS; MATTOS; DIERCKS, 2020). Além disso, o desafio está também em manter uma alimentação diferente do restante da família, participar de eventos sociais e é ainda maior quando a pessoa com diabetes é responsável pelo preparo da alimentação da família.

A questão financeira também pode ser uma barreira para a adesão a orientações e a implementação de uma alimentação saudável (REIS et al., 2020; BLASCO-BLASCO; PULG-GARCIA; PIAY et al., 2020). Associado a isso, a cultura desempenha importante influência nas escolhas alimentares (SOCIEDADE BRASILEIRA DE DIABETES, 2020). Segundo as Diretrizes da Sociedade Brasileira de Diabetes o plano alimentar necessita considerar além das necessidades nutricionais, as preferências, cultura, acesso aos alimentos, motivação e habilidades da pessoa com diabetes para mudança de comportamento (SOCIEDADE BRASILEIRA DE DIABETES, 2020).

O gradativo abandono do tratamento, principalmente alimentar, foi apontado pelos enfermeiros como um dos motivos de complicações e internações. Nos discursos 
dos enfermeiros há um movimento de culpabilizar a pessoa com diabetes por não seguir com as orientações alimentares, porém buscam entender a complexidade que é gerir uma doença crônica como a diabetes. Achado semelhante sobre o abandono foi descrito em estudo em decorrência de esgotamento da pessoa pela diabetes. Distanciar-se do tratamento resulta em um sentimento destrutivo de impotência, que pode se manifestar nas mais diversas formas, entre elas, hábitos alimentares inadequados, ausência de atividade física, monitoramento deficiente de glicose, não adesão às consultas de acompanhamento, entre outras (ABDOLI et al., 2019).

Há evidência de que imposições de dietas alimentares, sem um processo de negociação e participação da pessoa com diabetes, tendem a promover sofrimento e por esse motivo, sua desconsideração por parte dela (PERES et al., 2007). Os profissionais apresentaram tendência a culpar as pessoas com diabetes pelas dificuldades encontradas na adesão ao tratamento proposto (GAMA; GUIMARAES; ROCHA, 2017). Assim, sentimentos de medo do tratamento e culpa são gerados pela possível falta de cuidados prévios, principalmente medidas não farmacológicas, como a alimentação e o exercício físico (REIS et al., 2020).

$\mathrm{O}$ apoio emocional pode ser proporcionado pelo enfermeiro às pessoas com diabetes durante a hospitalização e é apontado como necessário para o processo de recuperação da saúde, e autogerenciamento da doença e tratamento (NIKITARA et al., 2019; PELTOLA; ISOTALUS; ÅSTEDT-KURKI, 2018). Esse apoio também necessita ser ampliado à família com a finalidade de construir vínculo e uma relação de confiança.

O acompanhamento contínuo de um profissional de saúde à pessoa com diabetes e a educação em saúde têm potencial para transformar essa realidade (REIS et al., 2020). Assim, "ações educativas realizadas em perspectiva dialogal, reflexiva e crítica podem ser instrumento efetivo para a formação de um conhecimento crítico”. Este possibilitará ampliar a compreensão dos indivíduos e a sua autonomia diante das condições de vida e saúde, no caso, o diabetes" (SOCIEDADE BRASILEIRA DE DIABETES, 2020, p.164).

Estudo de Arruda e Silva (2020) descrevem que ações coletivas para grupos de pessoas com diabetes que se encontram hospitalizadas são importantes e possíveis de serem realizados no hospital, que poderiam se beneficiar com o compartilhamento de experiências e conhecimento e, estímulo ao autocuidado. Além de grupos, outras estratégias para desenvolver ações educativas são, oficinas e palestras, considerando as características da pessoa, como idade, escolaridade, tempo de diagnóstico, aspectos psicossociais e culturais (SOCIEDADE BRASILEIRA DE DIABETES, 2020). Essas 
ações podem contar com profissionais de saúde das diversas áreas, além da enfermagem, nutrição, psicologia, serviço social, medicina, entre outros (ARRUDA; SILVA, 2020; SOCIEDADE BRASILEIRA DE DIABETES, 2020).

\title{
3.4 "Então essa é uma dificuldade também, perceber a adesão ao tratamento": manejode medicamentos
}

Para os enfermeiros, outro obstáculo vivenciado na assistência à pessoa com diabetes, corresponde ao manejo de medicamentos. O confronto entre o que os pacientes utilizavam como medicamento no domicílio, com o que recebem durante a hospitalização, principalmente quando há a inclusão da insulina como parte do tratamento medicamentoso, torna mais difícil o controle da glicemia no hospital por não querer fazêla. Tal achado é identificado no seguinte relato:

\begin{abstract}
Às vezes a gente tem paciente que está prescrito a insulina, mas ele não aceita fazer porque em casa ele não fazia. Eles dizem, "ah, mas eu só tomava comprimido em casa, não vou fazer insulina", aí está com a glicemia lá nas alturas. [...]. É, porque claro, é diferente do cuidado que eles têm em casa, paciente tomava uma metformina e uma glibenclamida e aí chega aqui não vai ser usado nenhum dos dois, vai ser usado o controle de HGT e a insulina se necessário. Normalmente esse é o esquema prescrito e, aí eles não aceitam essa mudança (E01).
\end{abstract}

Para os enfermeiros, o que desencadeou a internação hospitalar, foi porque os pacientes não seguem e cumprem com o que lhes é prescrito para uso no domicílio. Quanto ao uso de medicamentos estão relacionados, à automedicação, o não uso da insulina, o uso inadequado de insulina e hipoglicemiantes orais aumentando a dose, além da não administração da insulina quando poderiam fazê-la. O uso inadequado de medicamentos foi justificado pelo fato de que em situações específicas, como participação em eventos sociais com o aumento do consumo de doces aumentam também a dose do medicamento. A facilidade com que os pacientes com diabetes conseguem acessar os medicamentos na farmácia foi descrito como sendo um dos motivos que leva à automedicação. Estes achados são evidenciados nos seguintes relatos:

Muitas coisas que acontece, o paciente insulinodependente que não faz uso, de que não se administram a insulina, que a família administra. Quando vai fazer, relatos deles familiares, quando vai comer um doce ou vai para uma festa assim, faz mais insulina. Olha o risco! Entende? Mas não morreu, deu certo, continua fazendo, como se estivesse, empiricamente, tudo bem, está fazendo de forma correta e, não é (E09).

Falta de informação e, essa coisa também de usar medicações, essa automedicação, porque são medicamentos que tu podes comprar, até sem a tua receita. Se pegastes a primeira vez, às vezes na farmácia tu consegue pegar as outra vezes, até passar os seis meses (E015). 
A automedicação, o uso inadequado de hipoglicemiantes e a resistência em usar determinados medicamentos foi apontada pelos enfermeiros como um obstáculo durante a hospitalização. A resistência ao uso da insulina aparece como exemplo, podendo ser justificada pela forma de administração, os efeitos no corpo, e o custo. Nos discursos dos enfermeiros, o paciente entende que pode utilizar-se de mecanismos de compensação para normalização dos níveis de glicemia, se permitindo consumir alimentos que não lhe são indicados.

Há evidências de que o uso inadequado de medicamentos é decorrente da falta de orientação e informação sobre a diabetes e a correta utilização do tratamento que resulta no descontrole glicêmico, com oscilações entre episódios de hiperglicemia e hipoglicemia e, até mesmo em outras complicações (REIS et al., 2020; RODRIGUES; LIMA; SANTOS, 2015). Por outro lado, estudo desenvolvido em idosos com diabetes na Bahia identificou menor adesão, não proposital, aos medicamentos em decorrência do esquecimento e atraso nos horários das medicações. Essas atitudes no uso das medicações pode ser resultado das alterações cognitivas da idade (FERNANDES; DAMASCENA; PORTELA, 2019). Associado a isso, outra questão importante que necessita ser considerada pelo profissional é o gerenciamento de inúmeros medicamentos que fazem uso, polifarmácia, para a diabetes e comorbidades, o que pode comprometer o fluxo do tratamento e resulta em uma sobrecarga no cotidiano (MARTINS; MATTOS; DIERCKS, 2020).

Associado a falta de orientação e de informações, há outros obstáculos vivenciados pelas pessoas com diabetes como falta de acesso a medicamentos e insumos (seringas, agulhas, fitas reagentes e glicosímetros) que dificultam em manter o tratamento no domicílio. Há ainda situações que possuem uma menor condição econômica, e comprar medicamentos e insumos podem comprometer a renda familiar. Assim, o tratamento medicamentoso deixa de ser realizado, ou ocorre a reutilização de seringas e agulhas de maneira inadequada (REIS et al., 2020).

A educação em diabetes intra-hospitalar necessita ser desenvolvida, considerando que a pessoa com diabetes retornará para seu domicílio com a responsabilidade de tomar suas próprias decisões. Assim, durante o período de internação é necessário identificar "medidas de sobrevivência" segundo a necessidade individual, e entre estas medidas está conhecer os medicamentos tanto orais como injetáveis para a diabetes e o uso apropriado e manejo de agulhas e seringas (SOCIEDADE BRASILEIRA DE DIABETES; 2020) 
Estudos mostram que os enfermeiros possuem habilidades e competências para interagir com a pessoa com diabetes, acolhendo suas necessidades de saúde, crenças e desejos. Os enfermeiros podem desenvolver estratégias para conscientizá-los, promovendo o empoderamento e coduzilá-la para autogerir o tratamento (RODRIGUES; LIMA; SANTOS, 2015; NIKITARA et al., 2019).

\section{5 "O entendimento da própria família é bem difícil"}

Para os enfermeiros, um dos obstáculos está relacionado à família, a qual possui dificuldade de entender e aceitar o que está acontecendo. Este entendimento vai além de receber as informações, pois há um movimento da família para querer entender e aceitar para poder fazer. Tais achados são evidenciados nos seguintes relatos:

\footnotetext{
Então esse paciente já veio da UTI, da UTI ficou aqui conosco, então, era uma família bem humilde, e, eles não queriam entender, entende, acho até que eles entendiam, eles não queriam aceitar, sabe (E03).

O cuidado básico que tem que ter, eles [família] levam uma vida assim [sem cuidados]. Os pais já foram diabéticos, a família toda é diabética e, a alimentação e os cuidados todos, não são tão importantes para ele, no ponto de vista deles [família] (E02).
}

Para os enfermeiros há famílias que apresentam dificuldades em colaborar com a realização do tratamento durante a hospitalização. Para eles, a principal preocupação diante deste contexto se deve ao fato de que mesmo com todas as recomendações sobre $a$ dieta e o controle do que "devem comer", a família não às realiza. Essas ações são justificadas por acreditarem que essas exceções não influenciarão no tratamento. Diante disso, nas unidades de internação o "comer escondido" é um ritual frequente realizado pelos pacientes com a ajuda e aprovação dos familiares. Tais achados são identificados nos relatos a seguir:

Agora mesmo, tem um paciente que a gente está com ele ali, e ele come pastel de fora [do hospital] e aí os níveis dele tudo alterado. Ai a gente já falou, já pediu para o familiar, aí é complicado isso, a gente tenta (E07).

Então não é raro tu pegar um paciente diabético, insulinodependente já, comendo um pastel folhado. Não é raro isso acontecer aqui [unidade de internação da clínica], é muito diferente isso de setores fechados, onde o controle é maior. Porque às vezes tu está passando, fazendo exames nos pacientes e, tu te deparas com pacientes nos pertences dele comida, bolacha e tudo mais, entende.? Porque não é barrada a entrada [dos alimentos], então isso sim, isso é um problema muito grande para nós (E09).

É na verdade, no hospital não há problema porque eles recebem essa dieta, específica, mas eles acabam trazendo coisas de casa, que eles gostam. Os familiares trazem [os alimentos], que eles acreditam que não vai influenciar no tratamento. Eu acho que isso assim é o mais difícil assim, é seguir. Porque 
aqui, eles saem daqui e a gente sabe que eles não conseguem seguir em casa (E011).

A gente nota que às vezes até o entendimento da própria família é difícil, às vezes as orientações que dou, a comida, a nutrição, é mais assim para família, para eles entenderem. [...]. Porque as pessoas achavam assim, achavam não, entendiam que iam ter que mudar toda comida e, nunca mais iam poder comer doce, nunca mais, e de certa forma sim. Mas outras comidas que não são doces também tem grande quantidade de açúcar que elas teriam que reduzir e, elas não entendiam isso porque a comida não é doce, o arroz, a farinha, tudo isso que tem carboidratos complexos, e eles não entendem isso como açúcar, eles entendem isso como comida de sal. Então isso tudo é muito difícil para tu dizer pra pessoa e, aqui no hospital algumas pessoas a gente nota que ainda não conseguem compreender completamente o diagnóstico, outras não, ou já fazem isso a muitos anos, outras já lidam bem com isso.[...] (E013).

Para os enfermeiros a pessoa com diabetes e família tendem a associar o diabetes ao açúcar e doces, negligenciando o fato de que os carboidratos estão presentes em outros alimentos e que por isso necessitam consumir de forma consciente. Diante disso, como parte da assistência realizada pelos enfermeiros durante a internação, o aspecto alimentar ganha destaque, o qual envolve orientar e conscientizar acerca das mudanças e adaptações necessárias em seu cotidiano para melhorar sua condição clínica e qualidade de vida.

Quando os pacientes com diabetes são adolescentes ou jovens, a família tem maior dificuldade para aceitar a nova condição, uma vez que, se vêem "obrigados" a mudar hábitos de vida e ter restrições. Os enfermeiros ainda relataram que tentam mostrar a eles que podem levar uma vida "normal", porém necessitam desenvolver determinados cuidados para não desenvolverem complicações. Isto para os enfermeiros, foi visto como um processo difícil pois eles não mantêm vínculos com a família após a alta do hospital.

Os enfermeiros apresentam preocupações, por exemplo, pensar em estratégias para a conscientização - pessoa com diabetes-família - a fim de evitar complicações que levam a constantes (re)internações conforme o segmento de fala:

Ele é um paciente adolescente que quer viver uma vida normal e que não sabe viver de outra forma a não ser hoje, adolescente quer sair pra beber e pra comer, ele quer ter essa vida assim, eu sempre tento conversar e tá mais perto deles e mostrar que eles podem sim, que eles podem ter uma vida normal fora daqui, porque aqui eles vão ser controlados e muitas vezes a gente tem que controlar, porque aqui eles ficam comendo fora. E tento mostrar pra eles que sim, que pode ter, é que é uma questão de saúde, que são, é evitar que eles tenham consequências mais graves no futuro e principalmente pra familiar [...] As vezes até o próprio familiar que é resistente e muitas vezes até mesmo ao paciente né, porque muitas vezes ele não quer, ele sabe de tudo e ele não quer, são pacientes que, esse menino que eu estava te falando, que foi um menino que me chamou, porque como eu tô a pouco tempo aqui, eu acabo tendo menos ainda, agora vai vir a.... acho que tá há dois ou três anos aqui, mas foi um paciente que me chamou muita atenção e que era um paciente especificamente do diabete, que é um problema com a diabete. Ele foi um paciente que abriu fuga [fugiu] duas vezes. Porque quando você começa a restringir, a cuidar da alimentação e entra com as medicações, e ele tá um pouco bem, se recuperou, 
ele vai embora e aí ele volta de novo, ele voltou já, eu em quatro meses que eu estou aqui ele voltou duas vezes. Então as dificuldades são essas de entendimento (E02).

Há uma preocupação dos enfermeiros em conscientizar o paciente e seus familiares sobre as complicações que podem advir da não realização do tratamento, enquanto para o paciente, em especial o jovem, ele apenas quer viver sua vida "normal", semelhante aos outros jovens dos grupos aos quais pertence. Esta vida "normal” é representada pela liberdade de se socializar sem estar sendo controlado ou sendo refém da sua glicemia, como ocorre durante a hospitalização.

Para alguns enfermeiros a família é vista como um obstáculo por nem sempre "colaborar" no tratamento da pessoa com diabetes durante a hospitalização. Contudo, a família é considerada como fundamental no processo de autogerenciamento da diabetes e tratamento (SOCIEDADE BRASILEIRA DE DIABETES; 2020; BLASCO-BLASCO; PULG-GARCIA et al., 2020; REIS et al., 2020; SHAO et al., 2017).

Incluir a família nas ações de cuidado e de educação em saúde é essencial para o sucesso do tratamento da pessoa com diabetes. Estudos evidenciam que a contribuição do núcleo familiar pode resultar na melhora do controle glicêmico e no gerenciamento da diabetes (SHAO et al., 2017). O apoio da família tem papel significativo na alimentação e no autocuidado (REIS et al., 2020; SHAO et al., 2017). Isto porque o incentivo de pessoas próximas facilita a adaptação e coopera no tratamento (RODRIGUES; LIMA; SANTOS, 2015). Ademais, o apoio social por parte de familiares, amigos e comunidade pode fortalecer a determinação, crença e confiança na autogestão da condição de saúde e, consequentemente melhor qualidade de vida (SHAO et al., 2017).

As pessoas com diabetes são influenciadas pelas relações sociais relacionadas à família e amigos, sendo esses os principais determinantes do gerenciamento bemsucedido da doença (BLASCO-BLASCO et al., 2020). Experiências de familiares com diabetes podem ter uma influência negativa no manejo da doença em alguns casos, mas também foram vistas como um facilitador na medida em que podem melhorar a compreensão do paciente sobre a doença (BLASCO-BLASCO et al., 2020).

É necessário que durante a hospitalização o enfermeiro forneça informações à família, a quem na maioria das vezes é responsável pela pessoa com diabetes. É também a família como unidade social que, por meio de laços visíveis ou invisíveis, dará as soluções para esse cuidado (CASTRO-MEZA; PÉREZ-ZUMANO; SALCEDO-ÁLVAREZ, 2017). Para isso é necessário estratégias que reduzam os conflitos entre enfermeiros e 
família, promovendo um trabalho colaborativo entre os envolvidos, a fim de evitar fortalecer os vínculos e buscar desenvolver ações que possibilitem reduzir as complicações e melhorar a qualidade de vida da pessoa com diabetes.

\title{
3.6 "Mudança na rotina das refeições": a dieta no hospital
}

Outros obstáculos apontados pelos enfermeiros para à assistência de pessoas com diabetes diz respeito à mudança nos horários das refeições nas unidades de internação, troca da empresa terceirizada que fornecia as refeições, restrição do horário de funcionamento do serviço de nutrição terceirizado, insuficiente quantidade de alimento nas refeições para os pacientes e mudança na administração das dietas industrializadas.

Os enfermeiros relataram que as dietas preparadas tanto para refeições fornecidas para via oral, quanto para a utilização em sondas enterais tem ocasionado episódios de diarreia e desconfortos abdominais em pacientes. Isto foi descrito em decorrência da mudança da entrega de frascos fechados para abertos, implicando em uma manipulação adicional da dieta a ser distribuída. Estes achados podem ser descritos no seguinte relato:

\begin{abstract}
É uma das coisas que a gente mais verifica nessa troca do fornecedor das dietas que aconteceu agora recentemente é os casos de diarreia, eles se queixam de diarreia, aumento o caso de evacuação diárias, fica mais líquida, porque na verdade a dieta ela acaba fazendo com que a evacuação seja mais pastosa assim, e o que a gente verificou foi isso, as evacuações mais líquidas, aumento das evacuações, e eles tem se queixado muito de distensão abdominal e de tipo empachamento né, aquela sensação de tão saturados né, as vezes eles não querem nem fazer todas as dietas da noite, alguns pacientes até tem dietas intervaladas, duas horas faz dieta, para duas horas, faz duas horas e as vezes eles não querem a dieta das 2:00h da manhã, das 4:00h da manhã, das 6:00h da manhã, eles acabam dizendo que tão com aquela sensação de abdômen distendido sabe? É o que a gente mais percebe assim, esse tipo de alteração. [...]. Principalmente agora que eles tão manipulando, os frascos antes vinham fechados né, eram embalagens fechadas, seladas, e agora tipo eles tão trazendo em pequenas porções (E014).
\end{abstract}

Sendo o acompanhamento nutricional uma das condições importantes para o controle da glicemia, os profissionais enfermeiros estão atentos às dietas fornecidas, relacionando a mudança de fornecedor e, logística no preparo e entrega das dietas, aos eventos adversos apresentados pelos pacientes, que podem impactar diretamente na aceitação das refeições e, na recuperação da saúde. A segurança do paciente que faz uso de dieta enteral depende do processo, vigilância e avaliação contínua de profissionais da equipe de enfermagem, enfermeiro, técnico e auxiliar de enfermagem, demandando competências específicas para que evitar eventos adversos e desenvolver um cuidado seguro (CERVO et al., 2014). 
Para administração de terapia nutricional enteral é necessário um conjunto de cuidados que compreende a equipe de enfermagem, sendo que cada profissional, no cenário brasileiro, possui suas atribuições de acordo com regulamentação do Conselho Federal de Enfermagem (COFEN, 2014). Ainda, a Agência de Vigilância Sanitária determina um conjunto de boas práticas em terapia nutricional enteral que direciona a equipe de enfermagem, desde a indicação da dieta, prescrição, preparo, distribuição, administração, até o acompanhamento e monitoramento dos pacientes (ANVISA, 2000).

Adequar os horários para a realização do HGT, com a insulina e com a refeição é outra das dificuldades vivenciadas pelos enfermeiros que resulta no descontrole dos níveis glicêmicos dos pacientes. Esta adequação necessária é difícil de ser realizada, uma vez que, há, por vezes, atraso na entrega das refeições, aumentando o intervalo entre elas e, consequente aumento do consumo dos alimentos trazidos de fora do hospital pelos familiares ocasionando oscilações na glicemia, tanto hiperglicemia ou hipoglicemia. Para os profissionais enfermeiros, serem informados sobre mudanças institucionais que impactam diretamente na assistência, como mudança de horário de fornecimento das refeições é imprescindível para que se mantenha uma organização e planejamento no aprazamento dos hipoglicemiantes a serem administrados. Tais achados são evidenciados nos seguintes relatos:

A gente já tem que ter um olhar bem cuidadoso para estar aprazando nos horários certos a insulina, tanto a NPH como a regular, tem que saber o tempo que essa insulina vai fazer o efeito ou não. Agora mesmo no hospital a gente teve um problema que a gente trocou o pessoal que estava fornecendo a alimentação dos pacientes. Era uma empresa e aí agora passou para a empresa $\mathrm{X}$, eles estavam trazendo o café da manhã $9 \mathrm{~h}$ da manhã. Então a gente como enfermeira tem que se atinar, que a gente teria que muda o horário dos HGTS e das insulinas regulares, porque senão daqui a pouco está todo mundo fazendo hipoglicemia. Tu imaginas a gente fazer $6 \mathrm{~h} 30$ da manhã a insulina regular e aí até as $9 \mathrm{~h}$ o paciente não come nada porque o café ainda não chegou, ele vai fazer uma hipoglicemia (E012).

Inicialmente foi negativo, porque a gente não foi informado que mudaria o horário das dietas, se a gente tivesse sido informado obviamente a gente teria antecipado também o nosso aprazamento diferenciado, não foi informado. Então a gente foi pegá de surpresa e, aí três, quatro dias depois a gente viu que não, os pacientes estão dizendo que dieta está vindo mais tarde, então a gente vai ter que tomar uma conduta e trocar o horário dos aprazamentos. [...] A gente estava tendo problema de comunicação com os outros profissionais porque a gente tem folguistas [enfermeiro], que vem para cá, então a gente começou aprazar para mais tarde um pouquinho, por conta da dieta que estava vindo mais tarde, mas os folguistas não, então desregulava todo o que a gente tinha evoluído com o paciente. [...] (E012).

Para os enfermeiros, quando o paciente com diabetes interna é necessário que ele se adapte à dieta e aos horários das refeições. Contudo, esta adaptação pode ser "difícil", 
principalmente devido aos horários das refeições, sendo estes considerados como rígidos e como fatores que podem contribuir para a instabilidade da glicemia.

Vem uma dieta para o paciente e ele não se adapta as vezes a dieta ou as vezes, não vem no horário que ele costuma comer. Também o horário no hospital, ele é muito rígido, então, entra de novo no risco de glicemia instável, mas é, são várias questões, são várias etiologias para um mesmo diagnóstico (E05).

Então o paciente chega e não tá acostumado a comer naquele horário. E dentro do fluxo hospitalar tu recebe a dieta naquele horário, muitas vezes o jantar mesmo é praticamente a última refeição. Então os pacientes à noite, muitas vezes o paciente diabético recebe um lanche que muitas vezes na realidade dele não condiz com o, não consegue saciar dietamente, só com aquele lanche" (E08).

Ah, uma dificuldade que eu acho é uma questão se o paciente interna depois de um certo horário, a gente não consegue pedir para ele uma alimentação própria para patologia porque as vezes já não tem, como o serviço é terceirizado, eles tem até um certo horário para nos, para gente solicitar para eles, se não é o que tem, isso é uma dificuldade (E07).

A partir destes relatos, identifica-se a dificuldade que os enfermeiros encontram em articular a necessidade nutricional dos pacientes em horários em que o serviço de nutrição não está presente dentro do hospital, não sendo possível o acesso a refeição adequada no turno da noite, por exemplo, podendo haver abertura de exceções, fragilizando o trabalho de conscientização realizado.

Neste estudo identifica-se fragilidade na comunicação entre a gestão hospitalar e os enfermeiros das unidades de internação, quanto à dinâmica do serviço de nutrição terceirizado que parece influenciar na assistência de enfermagem à pessoa com diabetes. Os pacientes em uso insulina precisam de uma alimentação com quantidade específica de carboidratos, seguindo horários fixos, para evitar as hipoglicemias e descompensação dos níveis glicêmicos (SOCIEDADE BRASILEIRA DE DIABETES, 2020).

O enfermeiro como protagonista do cuidado tem sob sua responsabilidade o desenvolver a Sistematização da Assistência de Enfermagem, sendo esta um método potencial para resolução do problema, uma vez que planeja o trabalho em equipe e define os instrumentos que serão utilizados, sendo um trabalho conjunto e contínuo entre enfermeiros, equipe de enfermagem e gestão das instituições, o qual prioriza a qualidade da atenção realizada (SANTOS et al., 2016).

\section{Conclusão}

O estudo proporciona um panorama sobre os obstáculos vivenciados pelos enfermeiros na assistência às pessoas com diabetes hospitalizadas. Os enfermeiros 
vivenciam obstáculos para realizar a assistência ao paciente com diabetes durante a hospitalização, que estão relacionados ao próprio paciente, família, dinâmica do serviço de nutrição, gestão e, assistência de enfermagem do hospital. Para os enfermeiros, os pacientes que vivem com diabetes sofrem para além da dimensão física, tem o seu emocional e social afetados pelo adoecimento que influenciam no manejo do tratamento.

Esses obstáculos descritos diante da assistência de enfermagem no hospital ajudam a apreender a realidade de um contexto complexo, que envolve cuidados prévios que levaram a internação, cuidados durante a hospitalização e, a planejar a alta hospitalar. Esses obstáculos já foram identificados em outros estudos, contudo ainda se fazem presentes nos serviços da rede de saúde.

Compreender a assistência hospitalar ao paciente com diabetes como multidimensional, envolvendo orientações diversas sobre alimentação, medicação, estilo de vida, para o paciente e sua família, exige que o enfermeiro busque conhecer o paciente e, seu contexto familiar, social e cultural, em busca de abordagens que sejam efetivas e colaborem em uma maior participação ao tratamento, de forma a individualizar e valorizar o encontro profissional-paciente-família, almejando a criação de vínculos saudáveis e acolhedores.

Este estudo não pretende generalizar para o cenário nacional, todavia alguns destes achados podem ser aplicados a outros contextos, uma vez que, se compartilha o mesmo sistema de saúde no contexto brasileiro e legislações para a Enfermagem. Recomenda-se complementar esta perspectiva desenvolvendo estudos com as pessoas com diabetes e suas famílias sobre a atenção proporcionada no hospital, assim como outros profissionais de saúde da equipe e gestores.

\section{Referências}

ABDOLI, S. et al. Improving Diabetes Care: ShouldWeReconceptualize Diabetes Burnout?. The Diabetes Educator, Chicago, v. 45, n.2, p. 214-224, abr. 2019. Disponível em: DOI: 10.1177/0145721719829066. Acesso em: 05 jul. 2020.

AMERICAN DIABETES ASSOCIATION (ADA). Classification and Diagnosis of Diabetes: Standards of Medical Care in Diabetes-2020. Diabetes Care, Nova York, v. 43, n. 1, p.14-31, jan. 2020. Disponível em: https://doi.org/10.2337/dc20-S015. Acesso em: 20jun2020.

ANVISA. Agência Nacional de Vigilância Sanitária. Resolução no 63/2000 - Aprova o Regulamento Técnico para a Terapia de Nutrição Enteral. Diário Oficial da República Federativa do Brasil. Brasília, DF, 2000. 
ARRUDA, G. O. DE; SCHMIDT, D. B.; MARCON, S. S. Internações por diabetes mellitus e a Estratégia Saúde da Família, Paraná, Brasil, 2000 a 2012. Ciência \& saúde Coletiva, Rio de Janeiro, v. 23, n. 2, p. 543-552, fev.2018. Disponível em: https://doi.org/10.1590/141381232018232.23092015. Acesso em: 12 jul. 2020.

ARRUDA, C.; SILVA, D. M. G. V. A hospitalização como espaço para educação em saúde as pessoas com diabetes. Revista Cuidado é Fundamental Online, Rio de Janeiro, v. 12, p. 3745, jan./dez. 2020. Disponível em: http://dx.doi.org/10.9789/2175-5361.rpcfo. v12.6909. Acesso em: 20 jun. 2020.

BARDIN, L. Análise de Conteúdo. Tradução: Luís Augusto Pinheiro. São Paulo: Edições 70, 2016.

BLASCO-BLASCO, M.; PULG-GARCIA, M.; PIAY, N.; LUMBRERAS, B et al. Barreiras e facilitadores para o manejo bem-sucedido do diabetes mellitus tipo 2 na América Latina e no Caribe: uma revisão sistemática. PLoS ONE, São Francisco, v. 15, n. 9, p. e0237542, 2020. Disponível em: https://doi.org/10.1371/journal.pone.0237542. Acesso em: 07 mar. 2021.

BOGUN, M.; INZUCCHI, S. E. Inpatient Management of Diabetes and Hyperglycemia. ClinicalTherapeutics, Princeton, v. 35, n. 5, p. 724-733, maio .2013. Disponível em: DOI: 10.1016/j.clinthera.2013.04.008. Acesso em: 10 jul. 2020.

BOMMER, C. et al. Global Economic Burden of Diabetes in Adults: Projections From 2015 to 2030. Diabetes Care, Nova York, v. 41, n. 5, p. 963-970, maio. 2018. Disponível em: https://doi.org/10.2337/dc17-1962. Acesso em: 20 jul. 2020.

BRASIL. Ministério da Saúde. Conselho Nacional da Saúde. Resolução no 466/2012 - Aprova normas regulamentadoras de pesquisas envolvendo seres humanos. Diário Oficial da União. Brasília, DF, 2012.

BRASIL. Ministério da Saúde. Portaria no 1600, de 7 de Julho de 2011. Reformula a Política Nacional de Atenção às Urgências e institui a Rede de Atenção às Urgências no Sistema Único de saúde (SUS). Diário Oficial da União. Brasília, DF, 08 jul. 2011. Disponível em: https://bvsms.saude.gov.br/bvs/saudelegis/gm/2011/prt1600_07_07_2011.html. Acesso em: 05 mar. 2021.

CASTRO-MEZA, A.N.; PÉREZ-ZUMANO, S.E.; SALCEDO-ÁLVAREZ, R.A. La enseñanza a pacientes con diabetes: significado para profesionales de enfermería. Enferm. Univ., México, v. 14, n. 1, p. 39-46, mar. 2017. Disponível em:

http://www.scielo.org.mx/scielo.php?script=sci arttext\&pid=S1665-70632017000100039.

Acesso em: 07 mar. 2021.

CERVO, A.S.; MAGNAGO, T.S.B.S.; CAROLLO, J.B.; CHAGAS, B.P.; OLIVEIRA, A.S.; URBANETTO, J.S. Adverse eventsrelatedtothe use of enteral nutritionaltherapy. Revista Gaúcha de Enfermagem, Porto Alegre, v. 35, n.2, p.53-59, jun. 2014. Disponível em: https://doi.org/10.1590/1983-1447.2014.02.42396. Acesso em: 30 ago. 2020.

COFEN. Conselho Federal de Enfermagem. Resolução Cofen no 453/2014 - Aprova a Norma Técnica que dispõe sobre a Atuação da Equipe de Enfermagem em Terapia Nutricional. Diário Oficial da União. Brasília, DF, 2014.

COFEN. Conselho Federal de Enfermagem. Resolução Cofen no 564/2017 - Aprova o novo Código de Ética dos Profissionais de Enfermagem. Diário Oficial da União. Brasília, DF, 2017. 
FERNANDES, S. S. C.; DAMASCENA, R. S.; PORTELA, F. S. Avaliação da Adesão ao Tratamento Farmacológico de Idosos Portadores de Diabetes Mellitus Tipo II Acompanhados em uma Rede de Farmácias de Vitória da Conquista - Bahia. ID On-line Revista

Multidisciplinar e de Psicologia, Jaboatão dos Guararapes, v. 13, n. 43, p. 241-263, 2019. Disponível em: https://idonline.emnuvens.com.br/id/article/view/1522. Acesso em: 07 mar. 2021.

GAMA, C. A. P. DA.; GUIMARÃES, D. A.; ROCHA, G. N. G. Diabetes Mellitus e atenção primária: percepção dos profissionais sobre os problemas relacionados ao cuidado oferecido às pessoas com diabetes. Pesqui. prát. Psicossociais, São João Del-Rei, v. 12, n. 3, p. 1-16, jul./set. 2017. Disponível em:

http://pepsic.bvsalud.org/scielo.php?script=sci_arttext\&pid=S1809-

$89082017000300013 \& \operatorname{lng}=$ pt\&nrm=iso. Acesso em: 11 jul. 2020.

INTERNATIONAL DIABETES FEDERATION. IDF Diabetes Atlas, 9th ed. Brussels, Belgium: International Diabetes Federation, 2019.

MARAGNO, C. A. D.; LUIZ, P. P. V. Letramento em saúde e adesão ao tratamento medicamentoso: uma revisão da literatura. Revista de Iniciação Científica, Cricíuma, v. 14, n. 1, p. 01-14, 2016. Disponível

em:http://periodicos.unesc.net/iniciacaocientifica/issue/view/129/showToc. Acesso em: 16 ago. 2020 .

MARTINS, L. R.; MATTOS, M. B.; DIERCKS, M. S. Itinerário terapêutico de pessoas com diabetes mellitus tipo 2 que sofreram amputação de membro inferior: experiência do adoecimento. Revista de Atenção à Saúde, São Caetano do Sul, v.18, n.64, p. 72-84, abr./jun. 2020. Disponível em:

https://seer.uscs.edu.br/index.php/revista_ciencias_saude/article/view/6284. Acesso em: 07 mar. 2021.

MARQUES, M. B. et al. Intervenção educativa para a promoção do autocuidado de idosos com diabetes mellitus. Revista da Escola de Enfermagem da USP, São Paulo, v. 53, p. 01-08, dez. 2019. Disponível em: http://dx.doi.org/10.1590/s1980-220x2018026703517. Acesso em: 15 ago. 2020 .

MINAYO, M. C de S. Amostragem e saturação em pesquisa qualitativa: consensos e controvérsias. Revista Pesquisa Qualitativa, São Paulo, v. 5, n. 7, p. 01-12, abr.2017. Disponível em: https://editora.sepq.org.br/index.php/rpq/article/view/82/59. Acesso em: 16 out. 2020.

MULDER, B. C. et al. Effective nurse communication with type 2 diabetes patients: A Review. Western Journal of Nursing Research, Beverly Hills, v. 37, n. 8, p. 1100-1131, ago. 2015. Disponível em: https://journals.sagepub.com/doi/abs/10.1177/0193945914531077\#abstract. Acesso em: 11 ago. 2020.

MWILA, K. F.; BWEMBYA, P. A.; JACOBS, C. Experiences and challenges of adults living with type 2 diabetes mellitus presenting at the University Teaching Hospital in Lusaka, Zambia. BMJ Open Diabetes Research \&Care, Londres, v. 7, n. 1, p. 01-06, out. 2019. Disponível em: http://dx.doi.org/10.1136/bmjdrc-2017-000497. Acesso em: 20 jul. 2020.

NIKITARA, M. et al. O Papel dos Enfermeiros e os Facilitadores e Barreiras no Tratamento do Diabetes: Uma Revisão Sistemática da Literatura de Métodos Mistos. Behav. Sci., Basel, v. 9, n. 61, p. 01-16, jun. 2019. Disponível em: https://doi.org/10.3390/bs9060061. Acesso em: 07 mar. 2021. 
ORTIZ, L. DE. O. M. et al. Melhores práticas de enfermagem em educação em diabetes à criança hospitalizada: uma revisão integrativa. Revista Eletrônica de Enfermagem, Goiás, v. 19, p. 01-12, nov. 2017. Disponível em: http://doi.org/10.5216/ree.v19.45655. Acesso em: 09 jul. 2020.

PELTOLA, M.; ISOTALUS, P.; ÅSTEDT-KURKI, P. Patients' interpersonal communication experiences in the context of type 2 diabetes care. Qualitative Health Research, Thousand Oaks, v. 28, n. 8, p. 1267-1282, jul. 2018. Disponível em:

https://pubmed.ncbi.nlm.nih.gov/29542395. Acesso em: 15 ago. 2020.

PERES, D. S. et al. Dificuldades dos pacientes diabéticos para o controle da doença: sentimentos e comportamentos. Rev. Latino-Am. Enfermagem, Ribeirão Preto, v.15, n.6, p.1105-1110, dez. 2007. Disponível em: http://dx.doi.org/10.1590/S0104-11692007000600008. Acesso em: 07 mar. 2021.

REIS, P. dos. et al. Autocuidado e percepção do tratamento para o diabetes por pessoas em uso de insulina. Revista de Enfermagem da UFSM, Santa Maria, v.10, e60, p.1-20, jul. 2020.

Disponível em: https: 10.5902/2179769239880. Acesso em: 30 jul. 2020.

ROCHA, M. R. da. et al. Health literacy and adherence to drug treatment of type 2 diabetes mellitus. Escola Anna Nery, Rio de Janeiro, v. 23, n. 2, p. 01-07, fev. 2019. Disponível em: https://doi.org/10.1590/2177-9465-ean-2018-0325. Acesso em: 01 jul. 2020.

RODRIGUES, J.A.; LIMA, F.J.S.; SANTOS, A.G. Atuação do enfermeiro com pacientes com Diabetes Mellitus na melhoria da qualidade de vida. Rev Atenção Saúde, São Caetano do Sul, v.13, n.46, p.84-90, out./dez. 2015. Disponível em: https://doi.org/10.13037/ras.vol13n46.3102. Acesso em: 05 mar. 2021.

SANTOS, I. M. F. et al. SAE - Sistematização da Assistência de Enfermagem: Um guia para a prática. Salvador: COREN - BA, 2016.

SBD. Sociedade Brasileira de Diabetes. Diretrizes da Sociedade Brasileira de Diabetes: 2019-2020. São Paulo: Clannad, 2020. Disponível em: https://www.diabetes.org.br/profissionais/images/DIRETRIZES-COMPLETA-2019-2020.pdf. Acesso em: 07 mar. 2021.

SHAO, Y. et al. The effect of social support on glycemic control in patients with type 2 diabetes mellitus: the mediating roles of self-efficacy and adherence. Journal of Diabetes Research, Londres, v. 2017, p. 1-8, maio. 2017. Disponível em: https://doi.org/10.1155/2017/2804178. Acesso em: 07 mar. 2021.

Recebido em: 20 de novembro de 2021.

Aceito em: 25 de março de 2021. 